\title{
A COST EFFECTIVE TWO STAGE BIOGAS PROCESS FOR ORGANIC WASTE TREATMENT
}

\author{
Wenche Bergland ${ }^{1}$ \\ Finn Haugen ${ }^{2}$ \\ Rune Bakke ${ }^{1}$ \\ ${ }^{1}$ Department of Process, Energy and Environmental Technology, Telemark \\ University College \\ ${ }^{2}$ Department of Electrical Engineering, Information Technology and \\ Cybernetics, Telemark University College
}

\begin{abstract}
Effective energy recovery systems are required to handle organic waste produced at individual farms and industrial plants. High rate anaerobic digestion (AD) reactors (e.g. UASB) are efficient but they require low particle content in the feed, whereas manure and other relevant feeds contain a variety of particles.

A two stage biogas process consisting of a storage tank (ST) and a high rate AD reactor can meet the above challenges. The ST serves three purposes: Store the waste in a submerged suspension, disintegration and hydrolysis of the waste to produce dissolved substrate and separate a liquid without large particles as $\mathrm{AD}$ feed. A pilot system consisting of a $20 \mathrm{~L}$ hybrid AD reactor together with a $200 \mathrm{~L} \mathrm{ST}$ was constructed to test the two stage concept. The ST contained a filter system to extract only the liquid phase of the organic waste, which was solid waste from apple juice production. The AD liquid effluent was returned to the ST to maintain the systems liquid balance. A hydrogen sensor on the produced biogas was used to monitor the AD and thereby automatically control AD feeding for safe operation. Hydrogen set points of 20-40 ppm were tested.

The apple waste first underwent a natural conservation in the ST by production of organic acids and alcohol. The liquid feed to the AD extracted from the ST had a $\mathrm{pH}$ of 4.5 and a total organic concentration of $0-50 \mathrm{~g} \mathrm{COD} / \mathrm{L}$ through the months of operation reported. The temperature in the system was ambient; $18-23^{\circ} \mathrm{C}$. The production of biogas was $10-25$ litres per day, and the biogas methane content was maintained at $\approx 70(\mathrm{v} / \mathrm{v} \%)$. The hydrogen based $\mathrm{AD}$ feeding control operated well but with some limitations.
\end{abstract}

\section{KEYWORDS}

Anaerobic digestion; two stage AD; filter; apple waste; hydrogen sensor.

\section{INTRODUCTION}

Small and cost effective energy recovery systems are required to handle organic waste produced at small and medium scale farms and industrial plants. Methane production through anaerobic digestion offers significant advantages over other forms of organic waste treatment [1]. To keep the investment costs low, the reactor volume containing the anaerobic digestion

https://doi.org/10.15626/Eco-Tech.2010.057 
of the organic waste must be small and the digestion rate consequently high. High rate anaerobic digestion (HRAD) reactors (e.g. UASB) are natural choices since the volume of HRAD can be considerably lower than conventional mixed AD reactors.

Disintegration and hydrolysis of particles in the waste have very slow digestion rates, whereas the digestion rates of acidogenesis and acetogenesis in the liquid are high [2]. The digestion of waste containing particles therefore requires larger volumes to attain adequate methane production rate. In addition to this the HRAD require a feed with low particle content, whereas manure and many other relevant feeds contain a variety of particles in large quantities. The last digestion step, methanogenesis, is easily inhibited by a high acid content arriving from the former steps and is often carried out in a separate reactor.

To meet the above challenges a high rate anaerobic digestion (HRAD) reactor together with a storage tank (ST) containing a filter system to extract only liquid waste has been developed to investigate the potential of such two stage systems.

When a storage tank already exists, as for manure on most farms, reduced investment costs are likely by including one small reactor, instead of one or two large volume reactors required for the commonly used mixed reactor systems.

The waste is mixed with water and put in the storage in the two stage system investigated. The disintegration and hydrolysis will thereafter occur in the storage concentrating the soluble organic products in the liquid. Acidogenesis and acetogenesis can occur both in the storage and in the HRAD reactor. The methanogenesis preferably only occur in the HRAD reactor, but a gas collection should also be installed on the storage to collect methane if produced.

One challenge is to separate the liquid phase from the waste before feeding only the liquid phase into the reactor. Tests are ongoing on this separation to acquire a robust separation technique that may be different for different wastes.

Pure apple waste, from apple juice production, is used as substrate in this study, serving as a model substrate for farm and food processing wet organic wastes. According to Bouallagui $\mathrm{H}$. et al. [3] fruit and vegetable waste are rapidly acidified to volatile fatty acids (VFA), allowing a more constant feeding rate of the methanogenic second stage in two-stage systems. There is however also solid material in the acidified waste.

It is advantageous with a flexible reactor system where the production can be decreased and increased with changing demands for energy. To keep the operation costs low, the need for operators should be kept to a minimum. Monitoring the reactor by measuring the hydrogen content in the produced biogas is a cheap and easy to handle solution. Hydrogen is an intermediate and an end product of a digestion process, and using hydrogen as a system indicator for control purposes has been successfully implemented [4] with good results for control of the feed flow into a reactor. Limitations on using hydrogen for control purposes are also reported $[5,6,7]$. A manipulation of the feed flow based on the hydrogen concentration in the produced biogas is here implemented for automatic process control and evaluated for this purpose.

The main aim of the study is to evaluate the two stage process investigated as a potentially efficient biogas production scheme for wet organic wastes with many large particles. Secondary objectives are to investigate: Apple waste as feed for biogas production; Biogas production at ambient temperature; Biogas hydrogen content monitoring for process automation.

\section{METHOD}

The HRAD reactor and ST were operated as shown schematically in Figure 1. Liquid waste is pumped from the sieve in the ST, to the HRAD recirculation loop and into the bottom of the 
reactor together with recycled reactor liquid. The effluent is flowing back to ST to keep the liquid balance.

\subsection{Reactor and storage}

The reactor is a $20 \mathrm{~L}$ hybrid high rate $\mathrm{AD}$ reactor, with $17 \mathrm{~L}$ liquid phase and $3 \mathrm{~L}$ headspace. The lower half of the liquid volume is for suspended biomass inoculated with granular sludge. The volume in the middle section has plastic support medium for biofilm growth, and the top part is mainly for gas liquid separation.

An external circulation pump was used to pump the reactor content from the top to the bottom of the reactor for mixing and fluidization of granular sludge. The upward liquid flow was set at $1 \mathrm{~m} / \mathrm{h}$ to favour granular growth [8].

The ST is a 200 litre storage tank filled with apple waste and added water. A filtering device is mounted inside the ST. The filtering device consists of a $0.3 \mathrm{~m}$ long, $0.1 \mathrm{~m}$ diameter drainage pipe wrapped in a $1 \mathrm{~mm}$ opening mesh. A filter cake was expected to establish on this mesh when the liquid was withdrawn. To monitor the condition of the filter cake and the liquid inside the barrel, 3 pressure sensors were mounted on the filtering device inside the barrel (on the inside and outside surfaces of the sieve and $0.1 \mathrm{~m}$ outside the sieve). Clogging of the sieve or the filter cake would lead to changes in the pressure differences between the sensors. This system was tested by extracting liquid through the filter at rates from 0 to 4 litres / hour.

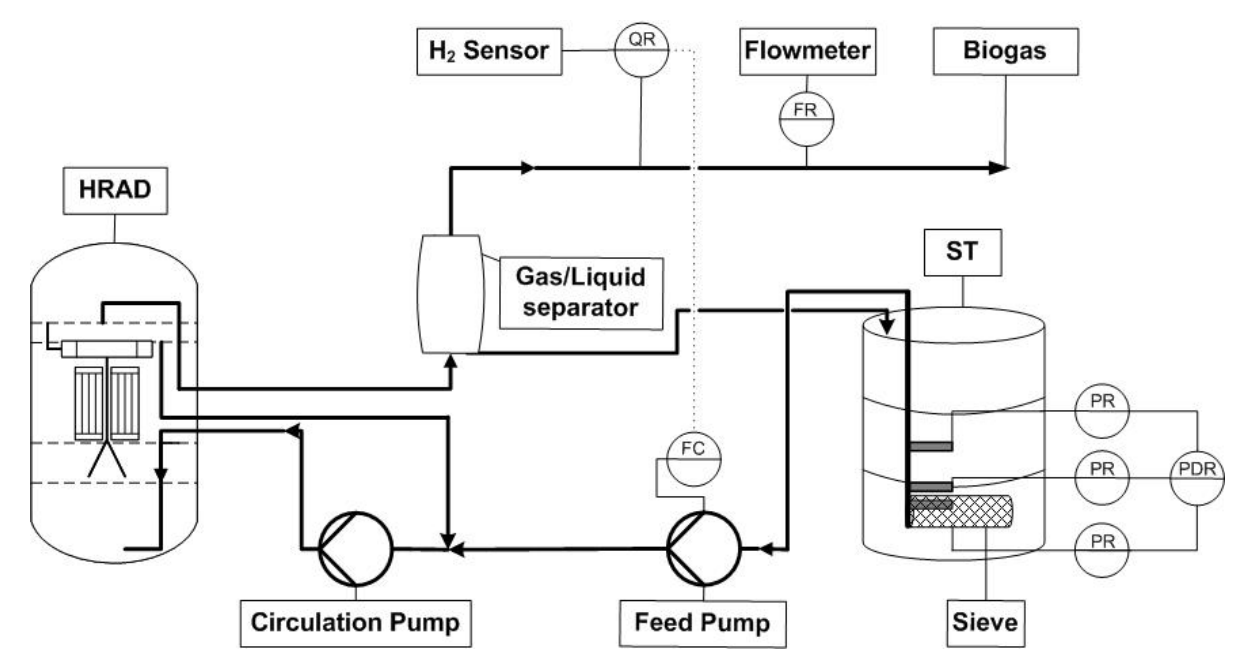

Figure 1. The high rate anaerobic digestion (HRAD) reactor and the storage tank (ST).

\subsection{Monitoring and control}

The amount of biogas produced was continuously measured using a gas clock flow meter Wilhelm Ritter KG. Bochum-Langendreer modified with 5 fixed inductive proximity switch sensors, SB3.5-E2-BU, Pepperl+Fuchs Group.

Off line measurements were done on filtered samples $(0.45 \mu \mathrm{m})$ for sCOD, VFA (acetate, propionate, butyrate, Iso-butyrate, valerate, Iso-valerate, caprionate, Iso-caprionate and heptanate), ethanol and $\mathrm{pH}$ of the feed and effluent of the reactor together with the gas composition of the produced biogas.

COD was measured with a HACH DR/2000 Spectrometer and analyzed according to US standard 5220D [9]. VFA and ethanol were measured with a Hewlett Packard Gas Chromatograph with a flame ionisation detector and a capillary column (FFAP $30 \mathrm{~m}$, inner diameter $0.250 \mathrm{~mm}$, film $0.50 \mu \mathrm{m}$ ). The oven was programmed to go from $100{ }^{\circ} \mathrm{C}$, hold for 
one minute, to $200{ }^{\circ} \mathrm{C}$ at a rate of $15^{\circ} \mathrm{C} / \mathrm{min}$, and then to $230{ }^{\circ} \mathrm{C}$ at a rate of $100{ }^{\circ} \mathrm{C} / \mathrm{min}$. The carrier gas used was helium at $23 \mathrm{~mL} / \mathrm{min}$. The injector and detector temperatures were set to 200 and $250{ }^{\circ} \mathrm{C}$, respectively. Gas composition $\left(\mathrm{CO}_{2}\right.$ and $\left.\mathrm{CH}_{4}\right)$ was quantified by gas chromatography (HewlettPackard 5890A) equipped with a thermal conductivity detector and two columns connected in parallel: Column 1, CP-Molsieve 5A (10 m x $0.32 \mathrm{~mm})$ and Column 2, CP-PoraBOND Q (50 m x $0.53 \mathrm{~mm})$. The gas carrier was argon at $3.5 \mathrm{bar}$ pressure. The oven temperature was kept constant at $40{ }^{\circ} \mathrm{C}$.

There was no temperature control of the reactor which operated at ambient (lab.) temperature, in the psychrophilic temperature range. The temperature varied between 18 and 24 degrees during the test period. Temperature was measured using a sensor mounted on the outside reactor wall and wrapped inside $5 \mathrm{~cm}$ thick insulation.

A control system was implemented to keep the biogas hydrogen content at a hydrogen concentration set point by manipulating the feed flow. Industry standard PI (proportional plus integral) control was used for hydrogen concentration control. Since increased hydrogen concentration implies increased feed flow, the feed flow was maximized indirectly by maximizing the set point of the hydrogen concentration.

The hydrogen concentration of the produced biogas was continuously measured by a hydrogen sensor type SENSOTOX 420 3HYT Sensotran with a measuring range of 0-2000 $\mathrm{ppm}$. The hydrogen set point was varied in the range from 20 to $40 \mathrm{ppm}$. If a higher production rate is required the hydrogen set point is increased and the feed is automatically increased.

The control system was implemented with LabVIEW running on a PC. The feed flow range demanded by the controller is in the very lower end of the range of the feed pump, causing problems with obtaining the demanded flow. Satisfactory pump control is obtained with Pulse-Width Modulation (PWM) where the pump is controlled as a binary (On/Off) device with the average flow being equal to the flow demanded by the controller. Different PWM cycle times in the range from 15 to 90 minutes were tried. The noisy $\mathrm{H}_{2}$ measurement signal was smoothed with a time-constant low pass filter with time-constant equal to 10 minutes before being connected to the controller.

\section{RESULTS AND DISCUSSION}

\subsection{Reactor and storage tank}

A robust filtering system to withdraw only the liquid phase for ST is required. A preliminary intensive test of the filter system, where the feed pump was running continuously for 5 hours at a constant rate of $0.8 \mathrm{~L} / \mathrm{h}$, showed a constant difference in pressure between the 3 pressure sensors inside the ST. This shows that the applied filter area is more than sufficient for the apple waste applied.

The rector was running for nearly a year producing biogas. The filtering system from the ST functioned well during this time, and extracted sufficient liquid waste at all time. During operation the pressures in the three locations inside the ST was constant, implying that the sieve and the filter cake never malfunctioned. This indicates a stable filter cake with an acceptable pressure drop and the sieve system tested is adequate for HRAD feed production from solid apple waste. The maximum rate successfully pumped through the ST filter system was $4 \mathrm{~L} / \mathrm{h}$ which correspond to a velocity of $0.47 \mathrm{~mm} / \mathrm{s}(1.7 \mathrm{~m} / \mathrm{h})$ through the filter.

The apple waste went through a natural conservation in the ST by production of organic acids and alcohol. The pH stabilized at 4.5 and the methanogenesis was therefore completely inhibited in the ST since a pH below approximately 6.2 results in inhibition of the methanogenesis process [10]. The organic content of the conserved liquid phase of the apple waste in ST was mainly acetic acid, ethanol and butyric acid. 
The maximum biogas production achieved in the HRAD was $1.5 \mathrm{~g} \mathrm{COD} / \mathrm{L} *$ day $(25 \mathrm{~L}$ biogas per day). The methane content in the biogas was $70 \%$.

The behaviour of the reactor from an early stage with high feed concentration until the biogas potential of the feed was used up is shown in Table 1 and Figure 2. They show the feed flow rate (reduced hydraulic retention time, HRT) at different times where the hydrogen concentration in the gas was in the range $30-40 \mathrm{ppm}$ and the temperature was $22-23{ }^{\circ} \mathrm{C}$. The control system was started at day 100 with a set point of $40 \mathrm{ppm}$ hydrogen which was changed to $30 \mathrm{ppm}$ at day 141 .

Table 1. Hydrogen concentration in the gas and HRT in the HRAD (temperature $22-23^{\circ} \mathrm{C}$ ).

\begin{tabular}{|cccc|}
\hline Days & $\begin{array}{c}\mathrm{H}_{2} \text { setpoint } \\
\mathrm{ppm}\end{array}$ & $\begin{array}{c}\mathrm{H}_{2} \\
\text { measured } \\
\mathrm{ppm}\end{array}$ & $\begin{array}{c}\text { HRT } \\
\text { (days) }\end{array}$ \\
\hline 44 & - & 30 & 35.4 \\
\hline 97 & - & 35 & 21.3 \\
\hline 119 & 40 & 40 & 12.1 \\
\hline 176 & 30 & 30 & 11.8 \\
\hline 205 & 30 & 30 & 11.6 \\
\hline 219 & 30 & 30 & 10.5 \\
\hline 238 & 30 & 30 & 2.8 \\
\hline 243 & 30 & 30 & 1.1 \\
\hline 251 & 30 & 30 & 0.2 \\
\hline 265 & 30 & 20 & 0.2 \\
\hline - sCOD g/L (VFA) in feed $\rightarrow-$ HRT (days) $\triangle$ B Biogas L/L*day
\end{tabular}

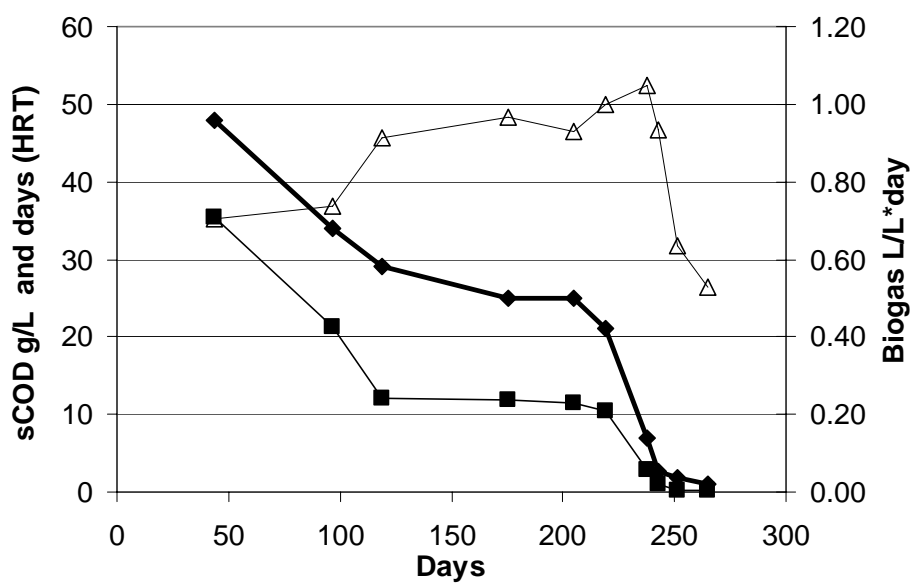

Figure 2. Biogas production, HRT and concentration ( $S C O D$ based on VFA) in the ST (feed) at measured hydrogen level 30-40 ppm and temperature $22-23{ }^{\circ} \mathrm{C}$.

An inspection of the biomass at the end of the experiment revealed suspended biomass but no granules. The reason for this can be the type of feed or the conditions in the reactor. Development of granular sludge should enable higher biogas production than was obtained here.

The flow of the feed into the reactor, and effluent back to the ST, resulted in a gradual dilution of the content in the ST. The $\mathrm{pH}, \mathrm{sCOD}$ and VFA of the liquid phase of the apple 
waste during the experiment are presented in Figure 3 and Figure 4. Directly measured sCOD and SCOD calculated based on VFA and ethanol measurements (Figure 4) are similar, implying that the organic feed composition to the HRAD was as shown in Figure 4 with no significant other organics. Ethanol was depleted faster than the VFA, suggesting that ethanol production dominated in ST initially while VFA production became more dominant later. The relative amounts of VFA remained relatively constant.

Biogas was also produced in ST when the $\mathrm{pH}$ increased above 6.2, which happened rather abruptly after $\sim 240 \mathrm{~d}$, when most of the SCOD production potential of the apple waste had been depleted (Figure 4). The apple waste had a pH of 4.5 before that, which was well below the critical $6.2 \mathrm{pH}$ level for methane production and therefore the biogas was only produced in the reactor at that time. In the end, when the $\mathrm{pH}$ went above about 6.5 , methane production started in the ST as well. The ST must in such situations have a collector system to collect this gas to avoid greenhouse gas emissions.

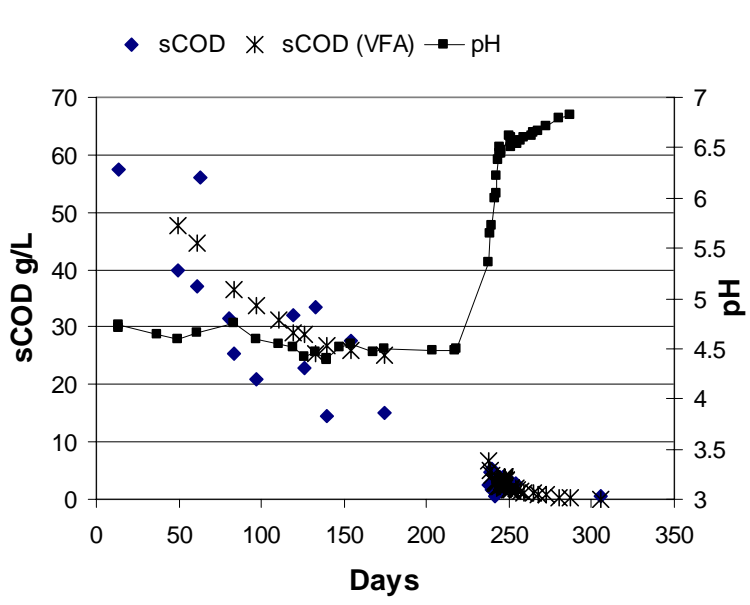

Figure 3. ST effluent $p H$, sCOD measured and sCOD $(V F A)$ calculated.

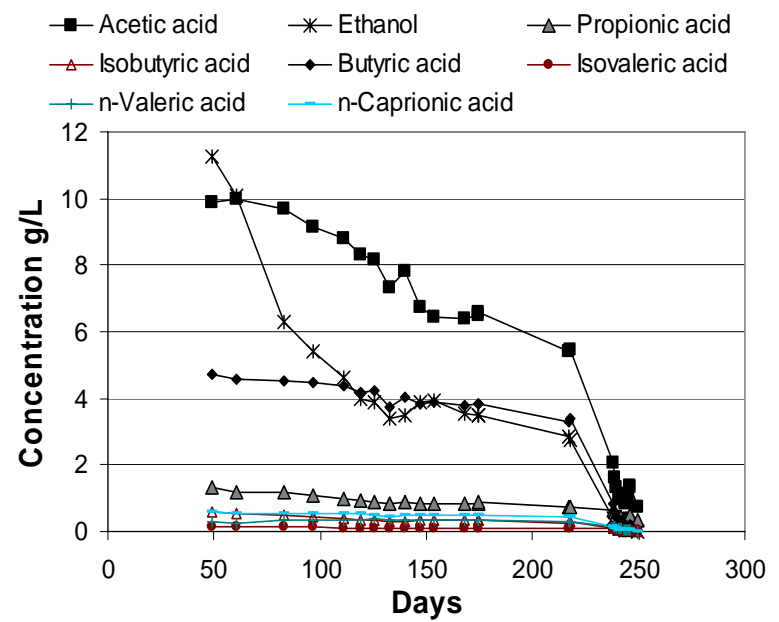

Figure 4. VFA content in the ST effluent.

\subsection{The control system}

The hydrogen concentration was controlled to reach a given concentration set point by manipulating the feed pumping rate. To test the response of the control system the set point of the hydrogen was varied stepwise. Changes in set point in the range 20 to $40 \mathrm{ppm}$ led to new hydrogen levels and new feed rates. Indirect feed flow control by maximizing the hydrogen concentration worked well when there were only small disturbances to the system. It showed a clear connection between the concentration level in the produced biogas and the feed rate. When increasing the set point of the hydrogen, the feed rate was increased and the hydrogen concentration was, after some fluctuating, setting in on the new set point value [11]. The concentration in the ST effluent was $30 \mathrm{~g}$ sCOD/L when the control system was implemented (day 100), and the control system worked well in maintaining high biogas production when the HRAD feed concentration decreased (Figure 2). The control system also prevented process overload, which can easily happen with such feed (low $\mathrm{pH}$, low alkalinity and high sCOD), by automatically reducing feed flow when hydrogen concentration was above set-point. Choosing the right set point is however not straightforward. 
There were some disturbances which clearly influenced the hydrogen based control system. Temperature fluctuations, feed concentration fluctuations and unintended introduction of a little air, all influenced the hydrogen concentration in the produced biogas. Hydrogen monitoring alone is, therefore, probably not sufficient for control purposes. Further research is needed to see how hydrogen can be part of a control system utilizing additional online measurements and reliable enough for full scale applications, especially for challenging feeds such as apple waste.

There was no temperature control in the system except the lab. room heating. The temperature during the test was in the range $18-24{ }^{\circ} \mathrm{C}$. Apparent effects of temperature changes within this range on the hydrogen concentration are seen in Figure 5.

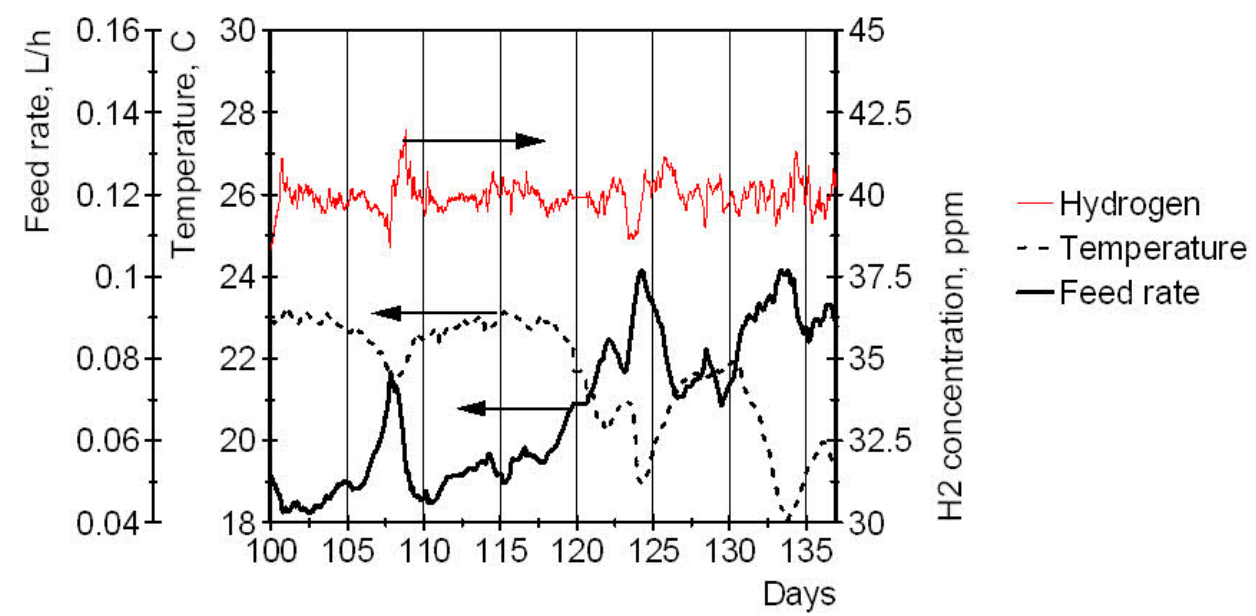

Figure 5. Change in feed flow with change in temperature.

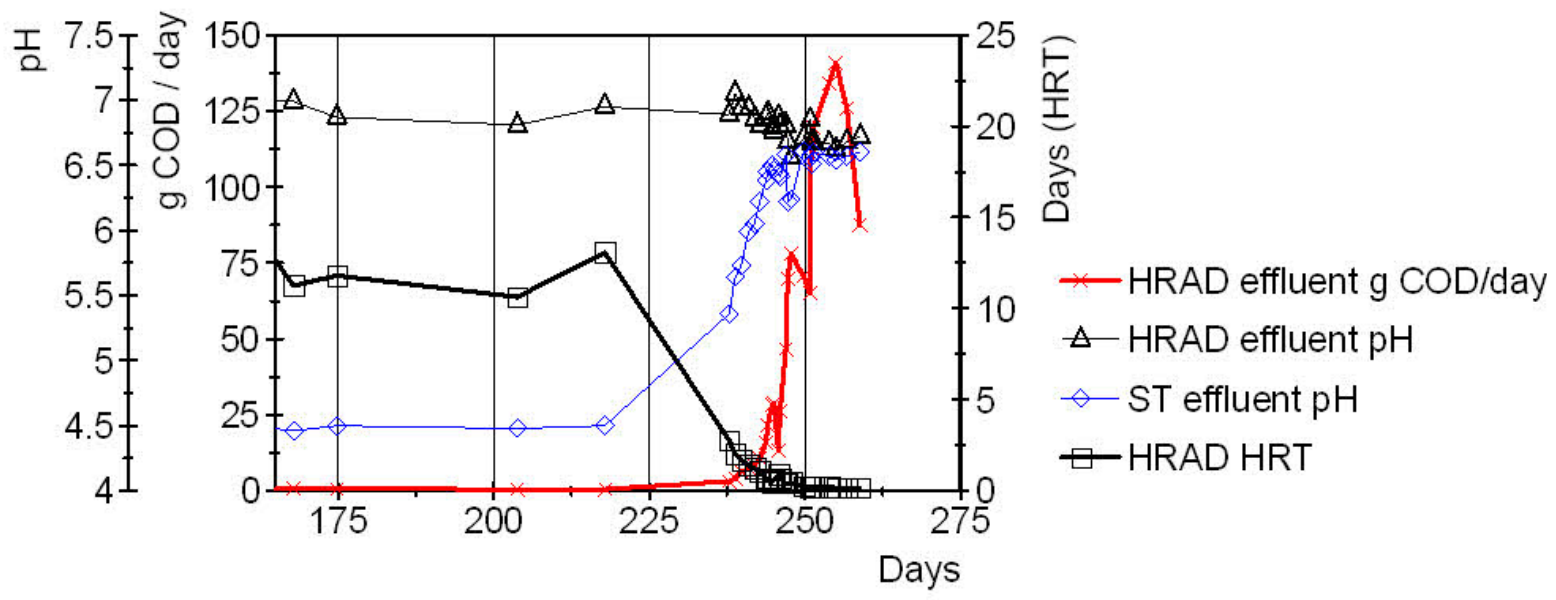

Figure 6. HRAD effluent sCOD, effluent $p H$ and HRT together with ST effluent $p H$.

The automatically controlled feed rate varies quite a bit but is slowly increased because of the decrease in the feed concentration, but in addition to that, an apparent effect from temperature reduction from $23{ }^{\circ} \mathrm{C}$ to $18{ }^{\circ} \mathrm{C}$ can be seen. The biogas production also increased. These changes resulted in a HRAD effluent $\mathrm{pH}$ decrease from 7.0 to $6.7( \pm 0.1)$ and reactor $\mathrm{pH}$ decrease from 6.9 to $6.4( \pm 0.1)$ in this period. The set point was therefore reduced from 40 to 
30 at day 141, to avoid low $\mathrm{pH}$ inhibition of methanogenesis. This shows that both a change in feed concentration and temperature in the reactor demand a new set point for the hydrogen to maintain stable operating conditions. This calls for a control system with higher performance, based on a mathematical model expressing the relation between temperature, feed composition and biogas hydrogen concentration. (Model-based control is the topic of future research on this reactor.) Alternatively, a sufficient temperature control could be used to maintain constant reactor temperature and avoid the effect of changing temperature.

The organic content of the ST waste was used up over time, forcing the control system to handle increasingly diluted feed. ST effluent concentration ranging from 30 down to $3 \mathrm{~g}$ $\mathrm{sCOD} / \mathrm{L}$ was handled well at a hydrogen set point of $30 \mathrm{ppm}$. When the organic content in the ST effluent became below $3 \mathrm{~g}$ sCOD/L (day 243), the HRT became too low for efficient production and the organic content in the HRAD effluent became to high, see Figure 6. At this point the biogas production rate started to decrease and the biogas methane content fell to $61 \%$.

Different intervals between feeding of the reactor, i.e. different PWM cycle times of the pump, showed an impact on the stability of the control system. Feeding intervals above 60 minutes led to hydrogen concentration instabilities, resulting in fluctuating feed rates. More frequent feeding gave more stable hydrogen concentration measurements and thereby more stable process operation. Small amounts of air inadvertently introduced in the reactor also gave an increase in the hydrogen concentration readings and subsequent reduction in the feed rate. Such disturbances should be avoided in a control system utilising hydrogen measurements.

\section{CONCLUSION}

The two stage process tested to produce biogas from a semi-solid wet organic waste worked well and appears to be an efficient way to recover energy from such wastes.

The filtering system in the waste storage tank, ST, functioned well extracting sufficient liquid feed for the high rate AD at all times.

The apple waste went through a natural conservation in the ST before the biogas production test started.

The biogas production had a maximum of $1.5 \mathrm{~g} \mathrm{COD} / \mathrm{L} *$ day.

The control system was sensitive to some process disturbance (air leaks, feed concentration variations, temperature changes and feed intervals) that should be studied further to make a more robust control system.

\section{REFERENCES}

[1] Ward A.J., Hobbs P. J., Holliman P.J., Jones D.L., 2008. Optimisation of the anaerobic digestion of agricultural resources. Bioresource Technology 99, 7928-7940.

[2] Batstone, D.J, Keller J., Angelidaki I., Kalyuzhnyi S., Pavlostathis S.G., Rozzi A., Sanders W., Siegrist H., Vavilin V., 2002. Anaerobic digestion model No 1 (ADM1) IWA Publishing, London.

[3] Bouallagui H., Touhami Y., Ben Cheikh R., Hamdi M., 2005. Bioreactor performance in anaerobic digestion of fruit and vegetable wastes. Process Biochemistry 40, 989-995.

[4] Rodriguez J., Ruiz G., Molina F., Roca E., Lema J.M., 2006. A hydrogen-based variable-gain controller for anaerobic digestion processes. Water Science \& Technology $54,2,57-62$. 
[5] Voolapalli, R. K., Stuckey D.C., 2001. Hydrogen production in anaerobic reactors during shock loads - influence of formate production and $\mathrm{H}_{2}$ kinetics. Wat.Res. 35, 7, 1831-1841.

[6] Pauss A., Andre G., Perrier M., Guiot S.R., 1990. Liquid-to-Gas Mass Transfer in Anaerobic Processes: Inevitable Transfer Limitations of Methane and Hydrogen in the Biomethanation Process. Appl Environ Microbiol. 56, 6, 1636-1644.

[7] Guwy A.J., Hawkes F.R., Hawkes D.L., Rozzi A.G., 1997. Hydrogen production in a high rate fluidised bed anaerobic digester. Wat. Res. 31, 6, 1291-1298.

[8] Metcalf \& Eddy, 2003, Wastewater Engineering. Fourth Edition. McGraw-Hill.

[9] APHA. Standard Methods for the Examination of Water and Wastewater. 19th edition. 1995. American Public Health Association/American Water Works Association/Water Environment Federation, Washington DC, USA.

[10] Cord-Ruwisch R., Mercz T.I., Hoh C.Y., Strong G.E., 1997. Dissolved Hydrogen Concentration as an On-Line Control Parameter for the Automated Poeration and Optimization of Anaerobic Digesters. Biotechnology and Bioengineering, 56, 6, 626634.

[11] Haugen F., Bergland W., Bakke R., Lie B., 2010, $\mathrm{H}_{2}$ concentration control of an experimental anaerobic biogas reactor. Venice. Third International Symposium on Energy from Biomass and Waste. 